\title{
Histochemical Analysis of Glycoconjugates in the Muzzle Skin of Egyptian water Buffalos (Bubalus bubalis) With Special Ref- erence to the Glandular Structure
}

\author{
Kassab Mohamed ${ }^{1}$, Abd elmaksoud Ahmed ${ }^{2}$, Hassanin Amin ${ }^{1}$, Yanai Tokuma ${ }^{3}$ \\ ${ }^{1}$ Department of Cytology and Histology, Faculty of Veterinary Medicine, Kafr El- sheikh University, Kafr El- \\ sheikh, Egypt. ${ }^{2}$ Department of Cytology and Histology, Faculty of Veterinary Medicine, Mansoura University, \\ Mansoura, Egypt. ${ }^{3}$ Department of Pathology, Faculty of Biological Science, Gifu University, Gifu, Japan.
}

\section{Summary}

In the present study, the distribution of various sugar residues in skin and nasolabial glands of the muzzle region of the Egyptian water buffalos were investigated by light microscopic histochemical methods, particularly lectin histochemistry. The epidermis and nasolabial glands were labeled with all lectins under investigation (LCA, ConA, PNA, $\mathrm{RCA}_{120}$, WGA, DBA, UEA-I, MAA, SSA and PHA$E)$ except the MAA and UEA-I which were negative in the epidermis and in the secretory acini respectively. Lectins labeling of the epidermis revealed the presence of mannosyl (LCA, ConA), galactosyl (PNA, RCA 120 ), N-acetyl-glucosamine (WGA), Nacetyl-galactosamine (DBA, PHA-E), L-fucose (UEA-1) and neuraminic acid (SSA). Interestingly, most layers of the epidermis exhibited different degrees of lectin labeling except the stratum cornium which showed negative reaction to all lectins. Similarly, the nasolabial glands revealed the presence of mannosyl (LCA, ConA), galactosyl (PNA, $\mathrm{RCA}_{120}$ ), N-acetyl-glucosamine (WGA), N-acetylgalactosamine (DBA, PHA-E) and neuraminic acid (MAA, SSA) while did not show any binding sites for L-fucose (UEA-I). The excretory ducts labeling were moderate to weak for all used lectins with two main features; firstly the present of basal striation resemble the striated ducts of salivary glands, secondly the lectin labeling was mainly confined to the luminal surface of their cells.

In conclusion, the complex carbohydrates with various sugar residues found in the epidermis and nasolabial glands of the muzzle region of buffalo may be involved in important functions, such as the preservation of humidity on the skin surface and the protection of the epidermis against physical damage or bacterial invasion. In addition, our results support the view of a salivary nature of the nasolabial glands in buffalo and emphasis that the functional significance of these glands type is not functionally different from that in other bovidae.
Key words: Glycoproteins, nasolabial glands, epidermis, muzzle, buffalos.

\section{Introduction}

It is now well established that, the specific body regions of mammals have undergone structural and functional adaptations that fit the animal for the biological requirements of its environment. In such, the maxillary lip of buffalo is modified to form extensive moist glandular nasolabial plate known as muzzle (Sarma et al., 2001). Anatomically, the surface of the buffalo muzzle showed hexagonal shaped areas separated from each other by grooves and in the center of these areas, small rounded openings of the nasolabial glands were seen (Farag, 2007).

The nature of the nasolabial glands is a matter of debate. Although, some investigators were described these glands as eccrine glands resembling those found in foot pad of carnivores (Meyer and Tsukise, 1989; 1995) and carpus of the pig (Calhoun and Stinson, 1981), others considered them as a combined form of both eccrine and apocrine glands and refers to them as intermediate gland in pig (Montagna and Yun, 1964). Additionally, these glands were previously explained as specialized salivary glands (Barone, 1976; Yasui et al., $2005 \mathrm{~b}$ ). The secretion of these glands and other glands on specific body regions have been shown to contain abundant glycoconjugates with various saccharides residues that have abroad biological significant to the skin function, such as, interspecies communication, the signaling of sexual activity, and water retention on the epidermal surface (Tsukise et al., 1983; 1988; Meyer and Bartels, 1989; Meyer and Tsukise, 1989; 1995; Yasui et al., 2003; 2004; 2005a; b).

Although, the nasolabial glands were studied in several animals including, cattle (Meyer and Tsukise, 1989), dog (Meyer and Tsukise, 1995), goat (Tsukise et al., 1988), Japanese deer (Yasui et al., 2005b), Japanese serow (Yasui et al., 2003), and 
pig (Tsukise et al., 1983), no data are to our knowledge available in buffalo. Therefore, this study was carried out to shed light on morphology and glycoconjugates histochemistry of the epidermis and glands of the muzzle region in the Egyptian water buffalo. Additionally, our study was focused on the species differences of the sugar residues of the skin and nasolabial glands of muzzle in an attempt to clarify if the functional significance of this gland type is fundamentally different from that in other bovidae or not?

\section{Materials and Methods}

The head of four buffalos were collected immediately after slaughtering from Kafr El-sheikh abattoirs, Egypt. Small samples of the muzzle region were dissected and fixed in Bouin's solution for 1824 hours at room temperature. The Bouin's fixed samples were extensively washed in $70 \%$ ethanol. The samples were dehydrated, cleared and embedded in paraffin wax using standard techniques. Sections $5 \mu \mathrm{m}$ thickness mounted on uncoated and coated slides with 3-aminopropyltriethoxysilane. For general morphology, selected slides were stained with hematoxylin and eosin.

\section{Conventional histochemistry:}

For general description of glycoconjugates, the slides were stained with periodic acid Schiff reagent (PAS) for neutral mucopolysaccharides and Alcian blue $(\mathrm{AB})$ at different $\mathrm{pH}(2.5,1.0$, and 0.5$)$ for acidic mucopolysaccharides (Pearse, 1985).

\section{Lectin histochemistry:}

Lectin binding sites were demonstrated by means of eight horse radish peroxidase (HRP) and two biotin labeled lectins. The slides were deparaffinized, then rehydrated in descending grades of ethanol until distal water. Endogenous peroxidase activity was blocked by incubation in $0.3 \%$ hydrogen peroxide $\left(\mathrm{H}_{2} \mathrm{O}_{2}\right)$ in methanol for 30 minutes at room temperature, thereafter the sections were incubated in $1 \%$ goat serum albumin (DAKO, USA) in phosphate buffer saline (PBS) for 20 minutes to minimize nonspecific staining. Subsequently, the slides were incubated with HRP/or biotinconjugated lectins (J Oil Mills, Tokyo, Japan) for one hour at room temperature. Lectins were generally used at concentrations of $20 \mu \mathrm{g} / \mathrm{ml}$ PBS pH 7.6 (Table 1). In case of biotinylated lectins, the slides were then incubated with streptavidin-biotin complex (ABC; Vector laboratories, Burlingame, USA) for 60 minutes at room temperature, and then rinsed in PBS (pH 7.2). After incubation, all sections (HRP/or biotin-labeled) were washed in PBS $(3 \times 5 \mathrm{~min}$.). Lectin binding sites were visualized using freshly prepared 3,3 'diaminobenzidine (DAB) solution (DAKO, USA) for 30 minutes at room temperature. Finally, the sections were washed in distal water $(3 \times 5 \mathrm{~min}$.$) , counterstained$ with Meyer's hematoxylin, dehydrated, and mounted with Mount-Quick (Daido Sangyo Co., Japan).

To examine the specificity of lectins labeling, control sections were prepared as follows: 1 ) the substitution of the lectin incubation medium with phosphate buffer saline; 2) the addition of hapten sugars to the respective lectin solution at the concentration 0.5M in PBS for one hour before labeling. The staining intensity was classified by two independent observers into 4 categories : no labelin (-), weak (+), moderate $(++)$, and strong $(+++)$.

\section{Results}

Histologically, the Planum nasolabialis (muzzle) of the Egyptian water buffalos was found to consist of epidermis, dermis and hypodermis (Fig. 1). The epidermis was generally composed of stratified squamous keratinized epithelium rested on highly vascular dense irregular connective tissue (dermis) containing some bundles of skeletal muscle fibers and nerves. The lobulated nasolabial glands were partially located in the deep portion of dermis and mainly in the hypodermis. Each lobule was composed of secretory acini and the duct system. The secretory acini were lined by pyramidal shape cells with centrally located round nuclei resemble those of the serous end-pieces of the salivary glands. Each lobule was drained by intercalated duct which connects to highly acidophilic duct resemble the striated duct of salivary glands (Figs. 1, 2). Although, the epidermis and the duct system were PAS negative (Fig. 3), a moderate PAS positive reaction was seen in the luminal portion the cells lining the secretory acini. In contrast, the $A B$ at different $\mathrm{pH}(2.5,1.0$, and 0.5$)$ did not reveal any positive staining neither in the skin nor in the nasolabial glands of the muzzle.

The glycoconjugates histochemistry of the epidermis showed variable reactivity in all lectins under investigation except MAA which was negative. The degree of labeling was differed according to the types of lectins and the layer of the epidermis (table 2). The basal cell layer was preferentially labeled with ConA, LCA, PNA, RCA ${ }_{120}$, WGA, DBA, SSA and PHA-E, while it was completely negative with UEA-I. Although, the stratum spinosum and stratum granulosum were bound to most of the investigated lectins (ConA, LCA, WGA, DBA, $\mathrm{RCA}_{120}$, SSA, UEA-I and PHA-E), the stratum granulosum exhibited no binding sites with PNA. Lectin-labeling of the epidermal cell layers was mainly cytoplasmic except for $\mathrm{RCA}_{120}$ and SSA where the binding sites were solely restricted to the cell boundaries of the adjacent cells. The stratum corneum was negative for all lectins. 
As a general role, the degree of labeling of the nasolabial glands was stronger in case of positive lectins than in the epidermis. The reaction of the secretory acini was differed from that of the excretory ducts (Table 2). The pyramidal cells of the secretory acini were strongly labeled with LCA, ConA, RCA ${ }_{120}$, WGA, DBA, SSA and PHA-E. Lectins-labeling was localized throughout the cellular cytoplasm of the pyramidal cell of the secretory acini except for the SSA and MAA where their binding sites were confined to the perinuclear region and to the cell boundaries and apical cell membrane of the pyramidal cells respectively. In contrast, PNA binding affinity was only seen in the luminal portion of the pyramidal cells while no binding sites for UEA-I was found in the secretory acini at all (Figs. 8, 9 and 10).

Labeling of the excretory duct cells was generally moderate to weak for all investigated lectins except ConA, $\mathrm{RCA}_{120}$ and WGA which showed a strong reaction and PNA which was negative. There were prominent features in the labeling of the ducts: firstly, the luminal secretions and the apical border of the epithelial cells lining the excretory ducts exhibited a strong positive reaction with all used lectins (Figs. 8, 9, 10, 11, and 12), secondly the reaction of the basal cell membrane enfolding of the striated ducts that resemble the striated duct of the salivary glands was clear by WGA, $\mathrm{RCA}_{120}$ and ConA (Fig. 12).

\section{Controls:}

None of the negative control sections that were performed by omission of the lectins showed any positive labeling. Similarly, all of the sections that were treated by pre-incubating lectins with corresponding hapten sugar inhibitor showed no labeling, except PNA, RCA 120 , WGA, MAA and SSA. With these sugars, there was significance diminution in labeling, but it was not completely abolished. This may result from all these sugars recognize more than one sugar binding partner and the inhibition experiments, the major haptenic sugar only was tested as inhibitor (Table 1).

\section{Discussion}

Lectins have a specific binding affinity for the sugar residues of glycoconjugates. Therefore they can be used as histochemical reagents to investigate the distribution of glycoconjugates in various tissue including epidermis and nasolabial glands (Tsukise et al., 1983; 1988; Meyer and Bartels, 1989; Meyer and Tsukise, 1989; 1995; Yasui et al., 2003; 2004; $2005 \mathrm{~b})$. In the present study, we have investigated the distribution of the sugar residues in the skin and nasolabial glands of the muzzle region in the Egyptian water buffalos by means of conventional and lectin histochemistry. Our results revealed that the epidermis and the duct system of the secretory acini were PAS-negative while a moderate PASpositive reaction was only observed in the luminal portion the cells lining the secretory acini. In contrast, the $\mathrm{AB}$ at different $\mathrm{pH}(2.5,1.0$, and 0.5$)$ did not reveal any staining neither in the skin nor in the nasolabial glands of the muzzle. These findings may indicate that the muzzle of the buffalos with its associated glands has not contained acidic glycoproteins but only neutral glycoproteins at the secretory end-pieces. These results are fairly consistent with the previous data of pig snout (Tsukise et al. 1983), and bovine muzzle (Meyer and Tsukise, 1989), wherever these studies have reported that, the secretory cells contain scant amount of acidic mucopolysaccharides but abundant amount of neutral mucopolysaccharides. On the contrary, other studies in goat (Tsukise et al., 1988), Japanese serow (Yasui et al., 2003), Japanese deer (Yasui et al. 2005b) have detected abundant amount of acidic and neutral mucopolysaccharides in the nasolabial glands of these animals. Regarding the saliva secretion in general, relatively high pH 8-9 (Majeed et al., 1970) may be explained by our findings. Such a $\mathrm{pH}$ range is in accordance with that known of bovine saliva (Lattmann, 1982). The actual secretion $\mathrm{pH}$ of the buffalos saliva is not known.

The lectin-labeling study of the epidermis revealed the presence of mannosyl (LCA, ConA), galactose (PNA, RCA), GlcNAc (WGA), GalNAc (DBA, PHAE), L-fucose (UEA) and sialic acid (SSA). The labeling is confined to most layers (basal, spinosum, and granulosum) in all lectins under investigation except the basal layer which is negative for PNA. The keratinized layer is negative to all lectins. This abundant amount of glycoconjugates containing mannosyl, galactosyl, glucosamine, galactosamine, fucosyl and sialic acid with moderate to strong labeling is present mainly in all layers. These results are fairly consistent with that of $\mathrm{Ya}$ sui et al. (2003) which described clear reaction to superficial, spinosum, granulosum and basal cell layers in Japanese serow, but unlike the previous work on the pig snout (Tsukise et al., 1983); in bovine muzzle (Meyer and Tsukise, 1989); the opossum snout (Meyer and Tsukise, 1990) and the snout of the wolf and domesticated dog (Meyer and Tsukise, 1995), whom described the reaction on the spinosum, granulosum and rarely in basal layer but did not describe any reaction to the superficial layer.

With regards to our results, the main saccharides residues are galactose and galactosamine in and in-between the epidermal cells, the presence of $\mathrm{N}$ acetyl galactosamine at the cell boundaries is an indicative of a role of these molecules in the regulation of movements of ions and fluids (Spicer and Schulte, 1992). Also to act as cementing and/or 
barrier substance, together with increasing the thickness of the epidermis, protecting the muzzle against mechanical and chemical hazards during normal biological function as feeding (Tsukise et al., 1983; Meyer and Tsukise, 1989; Yasui et al., 2003) although the lips of buffalo play a limited role in prehension of food as it depend on the large strong tongue in their feeding, in contrast to small ruminants, (May, 1970; Nickel et al., 1979; Farag, 2007).

The lectins-labeled study of the nasolabial glands secretory acini revealed the presence of mannosyl (LCA, ConA), galactosyl (RCA), glucosamine (WGA), galactosamine (DBA, PHA-E) and neuraminic acid (MAA, SSA) but negative for L-fucose (UEA). This result is not cooperated with that of Japanese serow in that more L-fucose was confirmed but agrees in the presence of more sialic acid (Yasui et al., 2003). The duct system of the nasolabial glands contain the same glycoconjugates, but the reaction is more pronounced in the luminal surface of their lining cells, while L-fucose which is negative for the acini are positive for the duct. The presence of fucosylated glycoconjugates may influence the transport functions in these ducts (Schulte et al., 1985; Meyer and Tsukise; 1990). Our results showed that the sialic acid was prominent at the boundaries of the secretory acini and luminal part of the duct lining cells and this finding was observed at the apical surface of such duct cells in the mandibular glands of certain mammals (Schulte et al., 1985; Menghi et al., 1992). The presence of sialoglycoconjugates confirms the view of a role of such substances in fluid and ion transport activities in bovine nasolabial glands (Wroble and Lindner, 1972; Yasui et al., 2003) and different salivary gland types (Davenport, 1971; Schulte et al., 1985). This may be significant in the rheology of such water-containing glycoproteins on the muzzle surface as the $\mathrm{N}$ acetyl neuraminic acid has a role in establishing cross links among glycoprotein molecules (Mandal and Mandal, 1990). The presence of high content of neutral glycoconjugates (mannosyl, galactosyl, glucosamine and galactosamine) may be important for water retention on the skin surface (Silberberg and Meyer, 1982; Tsukise and Meyer, 1987).

The scanning electron microscopic observation revealed that, the muzzle is formed of hexagonal shaped areas, separated from each other by grooves. In the center of these areas, small rounded openings of ducts of the nasolabial glands were existed. This pattern of arrangement keep the whole surface of Planum nasolabialis moist by the uniform distribution of the openings of the glands, at the same time, the grooves separating the hexagonal areas play a role in keeping the secretions over the epidermis (Farag, 2007). All these sugars liberated on the skin surface from their glycoprotein bases by microbial activities then gain the ability to inhibit the adherence of various bacteria and fungi to the outer cells of the epidermis as it does on the corneal cells (Meyer et al., 2000; 2001).

Regarding to the presence of fucosylated and sialoglycoconjugates in the intralobular ducts, together with the presence of striation in the basal border of these ducts, and due to enfolding of the basal membrane which is clear with (WGA, RCA 120) and (ConA) and the acidophelia of their cytoplasm, manifested by H\&E stain, this acidophelia is due to the presence of large number of mitochondria in the compartment made by basal membrane enfolding for transport of several ions and electrolytes. This could support our suggestion that, these ducts resemble the striated ducts of the salivary glands and play an important role in their secretion.

In conclusion, our results in the shape of secretory acini and the duct system especially the striated acidophilic duct and the appearance of clear basal striation, confirm the salivary nature of the nasolabial glands of buffalos and considered them as minor serous salivary glands but its secretion needs further investigation because the exact function is not clear. The presence of neutral glycoconjugates in the epidermis and glands of the muzzle region is of great significance especially in function as they keep the humidity of the skin and protect it from bacterial invasion and any physical damage.

\section{References}

Barone R. (1976): Anatomie compare des Mammiferes domistiques, vol 3 Lyon: Tixier and Fils.

Calhoun ML, Stinson AW. (1981): Integument. In: Dellman HD, Brown EM, editors. Text book of Veterinary Histology. 2nd ed. Phil adelphia: Lea and Febiger, 378-411.

Danguy A. (1995); Perspectives in modern glycolhistochemistry. Eur. J. Bas. Appl. Histo chem. $39,5-14$

Davenport HW. (1971):.Physiology of the digestive tract, 3rd ed. Chicago: Year Book Medical Publisher.

Debray H, Decout D, Strecker G, Spik J, Montreuil J. (1981): Specificity of twelve lectins to wards oligosaccharides and glycopeptides related to $\mathrm{N}$-glycosylprotiens. Eur. J. Bio chem., 117, 41-55.

Farag F. (2007): Some morphological studies on the oral cavity of buffalo. Dissertation, De- 
partment of Anatomy, Histology and Embryology, Fac. Vet. Med., Kafr Elsheikh Univ., Egypt.

Lattmann J. (1982): Untersuchungen uber den Elektrolutgehalt von speichel, serum und pansensaft gesunder sowie an labmagen verlagerungsbedingter storung ingestapassage erkranter Rinder. Diss. Vet. Med. Hannover..

Majeed MA, Zaidi IH, Ilahi A. (1970) :The nature of nasolabial gland secretion (NLGS) in large domestic ruminants. Res. Vet. Sci., 11, 407-10.

Mandal c, Mandal C. (1990): Sialic acid binding lectins. Experientia , 46, 433-41.

May ND. (1970): The anatomy of the sheep, a dissection manual. 3rd ed. Univ. of Queens land press..

Menghi G, Accili D, Scocco P, Materazzi G. (1992): Sialoglyco-derivatives of bovine submandibular gland identified in situ by histochemical techniques combined with lectins. Histochemistry; 97, 397-403.

Meyer W, Bartels T. (1989): Histochemical studies on the eccrine glands in the foot pads of the cat. Basic Appl. Histochem., 33, 21938.

Meyer W, Tsukise A. (1989): Histochemistry of glycoconjugates in the skin of bovine muzzle, with special reference to the glandular structure. Acta Anat.,136, 226-34.

Meyer W, Tsukise A. (1990): Structural and carbohydrates histochemical aspects of the snout skin of the opossum, Didelphis virginiana. Kerr. Zool. Sci., 7, 923-31.

Meyer W, Tsukise A. (1995); Lectin histochemistry of snot skin and foot pads in the wolf and the domesticated dog (Mammalia canidae). Ann Anat. 177, 39-49.

Meyer W, Bollhorn M, Stede M. (2004): Aspects of general antimicrobial properties of skin secretions in the common seal Phoca vitulina. Dis. Aquat. Organ. 41, 1, 77-79.

Meyer W, Neurand K, Tanyolac A. (2001): General antimicrobial properties of the integument of the fleece producing sheep and goats. Small Rumin. Res., 41, 181-90.

Montagna W, Yun JS. (1964): The skin of the do mestic pig. J. Invest. Derm., 43,11-21.

Nickle R, Shummer A, Seifrle E. (1979): Digestive system (mouth and pharynx, general and comparative). Text book of the viscera of domestic mammals. 2nd ed, Verlag Paul parey, Berlin, Hamburg.

Pearse A. (1985): Histochemistry, theoretical and applied, 4th ed, vol 2. Churchill Livingstone, Edinburg.

Pereira MC, Pereira AAC, Hanemann JA. (2007): Immunohistochemical profile of canalicular adenoma of the upper lip: a case report. Med. Oral. Pathol. Oral. Cir. Bucal.,12,1-3.

Sarma, M, Sarma K, Bhattachary M. (2001): Com parative Anatomy of the lips of mithun, yak and zebu. Indian Vet. J.,78, 513-14.

Schulte BA, Spicer SS, Miller RL. (1985): Lectin histochemistry of secretory and cellsurface glycoconjugates in the ovine submandibular gland. Cell Tissue Res., 240, 57-66.

Silberberg A, Meyer FA. (1982): Structure and function of mucus. In: Chantler EN, Elder $\mathrm{JB}$, Elestein $\mathrm{M}$, editors, Mucus in health and disease, Vol 2, New York,Plenum Press, 53-73.

Spicer SS, Schulte BA. (1992): Diversity of cell glcoconjugates shown histochemically: a perspective. J. Histochem. Cytochem.; 40: 1-38.

Tsukise A, Meyer W, Schwarz R.(1983): Histochemistry of complex carbohydrates in the skin of the pig snout, with especial refer ence to eccrine glands. Acta Anat., 115, 141-50.

Tsukise A, Meyer W. (1987): Histochemical analysis of carbohydrates in the scrotal skin of the horse, with special reference to glandular appendages. Zool. Anz. , 219 ,12940.

Tsukise A, Fujimori O, Yamada K. (1988): Histochemistry of glycoconjugates in the goat nasolabial skin with special reference to eccrine glands. Acta Anat., 132: 150-58.

Wrobel KH, Lindner E. (1972): Die Histotopochemie und Ultrastrukture der Glandulae nasolabiales des Rinders. Berl. Munch. Tierarztle. Wschr. 85: 480-84

Yasui T, Tsukise A, Nara T, Habata I, Meyer W. (2003): Histochemistry of glycoconjugates in the nasolabial skin of Japanese serow (capriconis crospus, Artiodactyla, Mammalia) with special reference to glandular structure. Eur. J. Morphol., 41, 43-51.

Yasui T, Tsukise A, Meyer W. (2004): Histochemical analysis of glycoconjugates in the eccrine glands of the raccoon digital pads. Eur. J. Histochem., 48, 393-402.

Yasui T, Tsukise A, Meyer W. (2005a): Ultracytochemical demonstration of raccoon (Procyon Lotor). Anat. Histol. Embryol. 34: 5660.

Yasui T, Tsukise A, Fukui K, Kuwahara Y, Meyer W. 2005b; Histochemical localization of complex carbohydrates in the nasolabial glands of the Japanese deer (Cervus Nippon yakushimae). Acta Histochem. 48: 187- 98. 


\begin{tabular}{|c|c|c|c|c|}
\hline Lectin* & Name & Sugar binding specificity & Binding & Inhibitor sugar \\
\hline CONA & Concanavalin $\mathrm{A}$ & $\alpha-D-M a n, \alpha-d-G l c$ & $\mathrm{HRP}$ & $\begin{array}{l}\text { Man, a-methyl D- } \\
\text { glucoside }\end{array}$ \\
\hline DBA & $\begin{array}{l}\text { Dolichos biflorus ag- } \\
\text { glutinin }\end{array}$ & GalNACa1-3GalNAC & HRP & a-D-GalNAC \\
\hline LCA & Lens culinaris & a-Man & HRP & a-Man \\
\hline PHA-E4 & Phaseolus vulgaris & D-GalNAC & HRP & D-GalNAC \\
\hline PNA & Peanut agglutinin & Gal $\beta 1-3 G a l N A C$ & HRP & $\begin{array}{l}\beta-D-G a l, \alpha- \\
\text { Lactose }\end{array}$ \\
\hline $\mathrm{RCA}_{120}$ & $\begin{array}{l}\text { Ricinus communis ag- } \\
\text { glutinin } 120\end{array}$ & Gal $\beta 1-4 G \mid c N A C$ & HRP & $\beta-D-G a l$ \\
\hline UEA-1 & $\begin{array}{l}\text { Ulex europaeus agglu- } \\
\text { tinin } 1\end{array}$ & $\alpha-L-F u c$ & HRP & $\alpha-L-F u c$ \\
\hline WGA & Wheat germ agglutinin & $\beta-D-G l c N A C$ & HRP & $\beta$-D-GlcNAC \\
\hline SSA & $\begin{array}{l}\text { Sambucus sieboldiana } \\
\text { agglutinin }\end{array}$ & Sia a2-6Gal/GalNAC & Biotin & Lactose, NeuNAC \\
\hline MAA & $\begin{array}{l}\text { Maakia amurensis ag- } \\
\text { glutinin }\end{array}$ & Sia a2-3Gal-4GluNAC & Biotin & Lactose, NeuNAC \\
\hline
\end{tabular}

Table 1: The lectins and their sugars binding specificities

Gal: Galactose, GalNAC: N-acetylgalactosamine, Glc: glucose, GlcNAC: N-acetylglucosamine, Fuc: fucose, Man: mannose, Sia: Sialic acid, NeuNAC: Neuraminic acid, HRP: horse radish peroxidase.

\begin{tabular}{|c|c|c|c|c|c|}
\hline Lectins & $\begin{array}{l}\text { Epide } \\
\text { Basal }\end{array}$ & upper & boundaries & $\begin{array}{l}\text { Gland } \\
\text { Acini }\end{array}$ & duct \\
\hline WGA & +++ & +++ & +++ & +++ & ++ \\
\hline UEA-1 & - & + & - & - & -/+ Luminal \\
\hline $\mathrm{RCA}_{120}$ & ++ & ++ & +++ & ++++ & ++ \\
\hline PNA & +++ & + & ++ & -/+ Luminal & -/+ Luminal \\
\hline LCA & ++ & ++ & - & +++ & $+/++$ Luminal \\
\hline CONA & ++ & ++ & +++ & +++ & +++ \\
\hline PHA-E4 & +++ & +++ & +++ & +++ & +++ \\
\hline DBA & ++ & ++ & +++ & +++ & + \\
\hline SSA & +++ & ++ & +++ & + & -/+ Iuminal \\
\hline MAM & - & - & - & + & $+/++$ luminal \\
\hline PAS & - & - & - & -/+ Luminal & - \\
\hline AB 2.5 & - & - & - & - & - \\
\hline AB 1.0 & - & - & - & - & - \\
\hline AB 0.5 & - & - & - & - & - \\
\hline
\end{tabular}

Table2: Histochemical activity in both the epidermis and glands of muzzle region in buffalo 

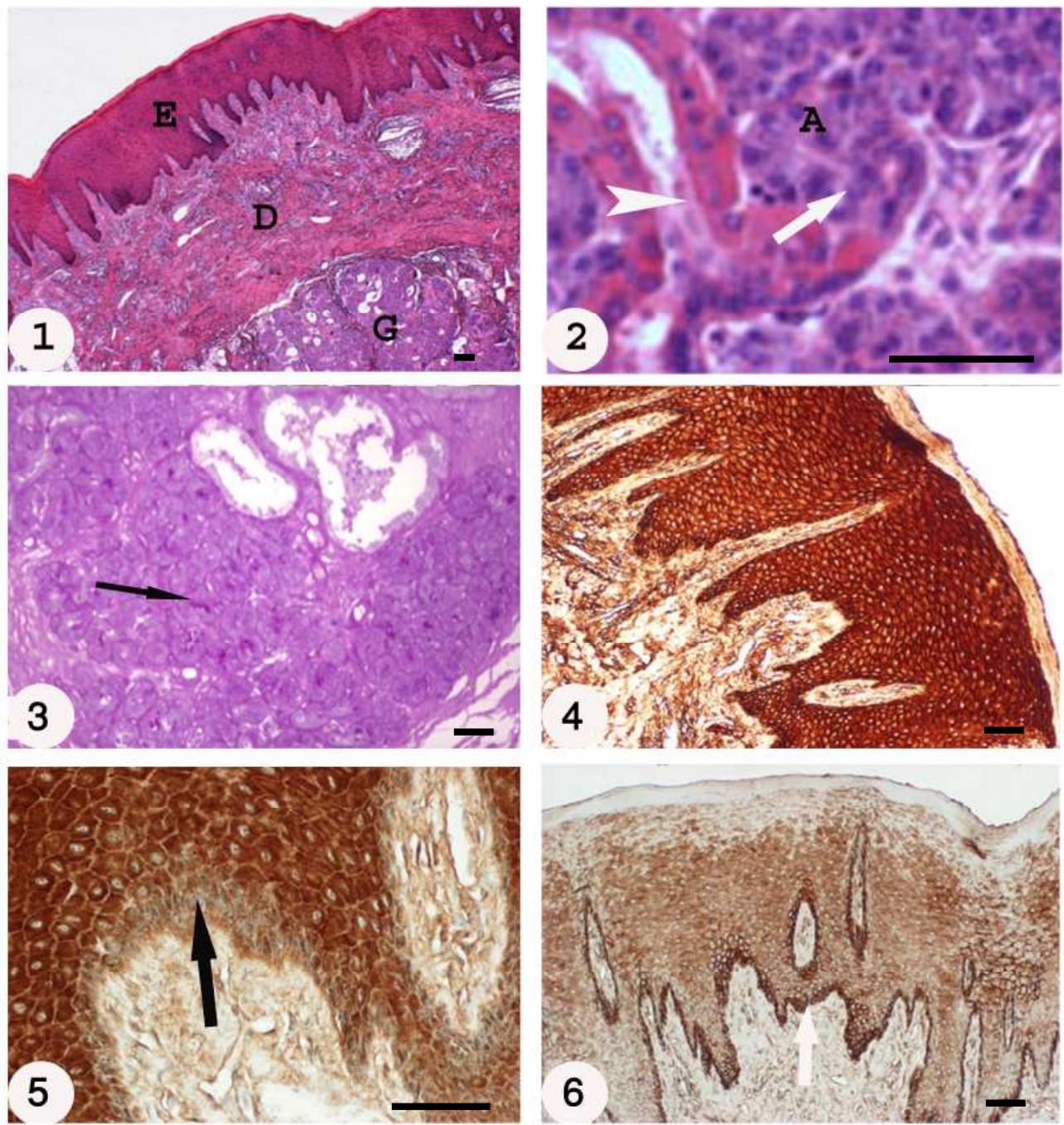

Fig. 1: General structure of the muzzle region showed epidermis (E), dermis (D), and hypodermis containing nasolabial glands (G). H\&E X20.

Fig. 2: Nasolabial glands with higher magnification showed its acini (A), intercalated duct (arrow), and striated duct (arrow head). H\&E X 400.

Fig.3: PAS reaction was restricted to the luminal surface of some acini (arrow). PAS X100.

Fig. 4: RCA 120 lectin reactivity to the epidermis. $X 40$.

Fig. 5: UEA lectin negative for the basal layer (arrow) and positive to the remaining layers in the cytoplasm only not cells boundaries. X 200 .

Fig. 6: PNA positive lectin in the basal layer of the epidermis and weak in the above layers. $X 40$. 

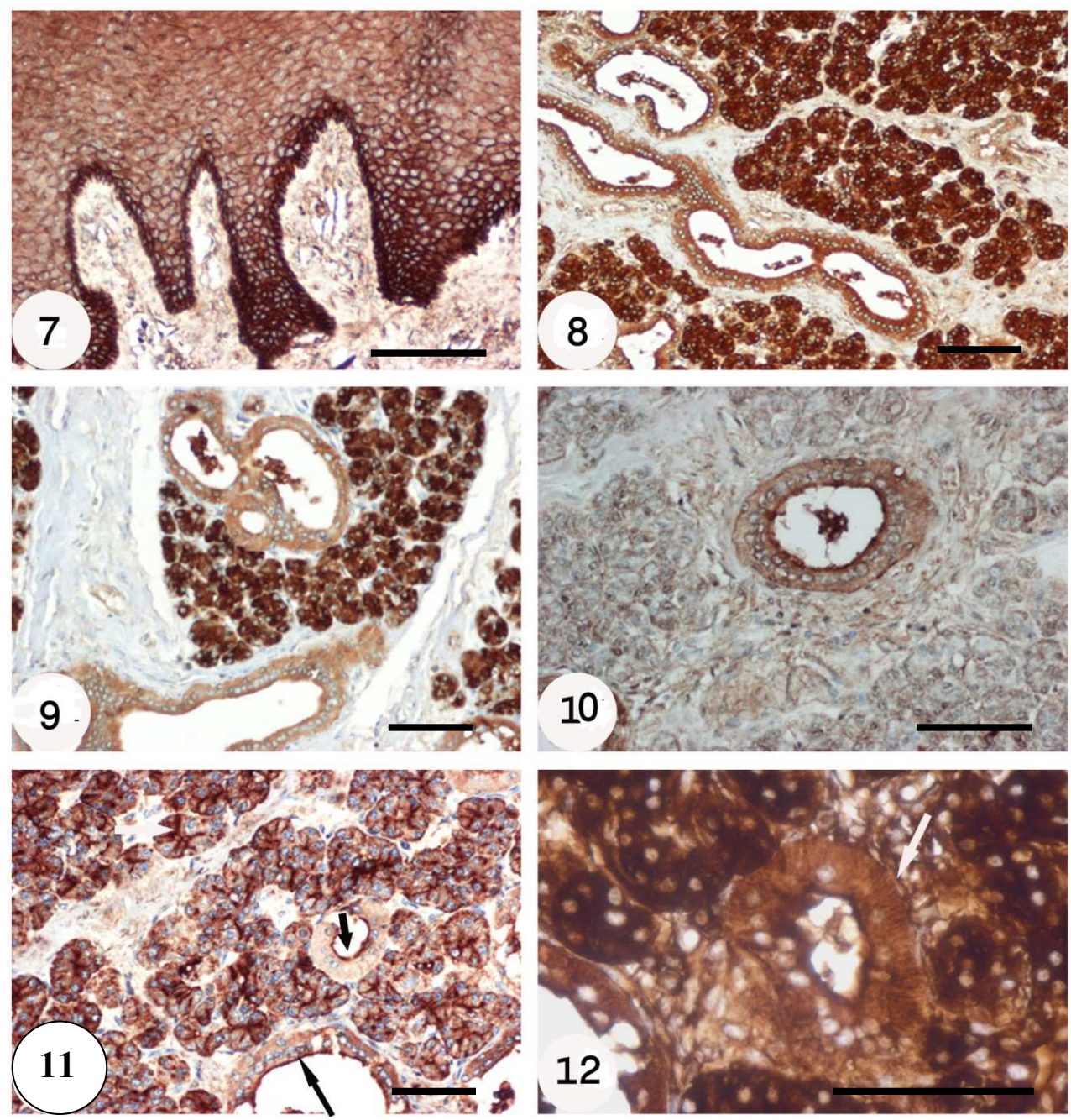

Fig. 7: SAA high reactivity in the basal layer and boundaries between cells and moderate reaction to the above layers. X200.

Fig. 8: ConA reaction to the nasolabial glands. $X 100$

Fig. 9: DBA reaction to the nasolabial glands. $X 100$.

Fig.10: UEA negative reaction to the secretory acini and positive for the duct. $X 200$.

Fig. 11: MAM positive reaction to the boundaries of secretory acinar cells and the duct (arrow) of the nasolabial glands. X 100 .

Fig. 12: RCA120 positive nasolabial glands, with prominent basal striation of the striated duct (arrow). X 800 .

J. vet. anat. 